\title{
Mode degeneracy due to vortex core removal in magnetic disks
}

\author{
F. Hoffmann, ${ }^{1}$ G. Woltersdorf, ${ }^{1}$ K. Perzlmaier ${ }^{1}$ A. N. Slavin, ${ }^{2}$ V. S. Tiberkevich, ${ }^{2}$ A. Bischof, ${ }^{3}$ D. Weiss, ${ }^{1}$ and C. H. Back ${ }^{1}$ \\ ${ }^{1}$ Department of Physics, Universität Regensburg, 93040 Regensburg, Germany \\ ${ }^{2}$ Department of Physics, Oakland University, Rochester, Michigan 48309, USA \\ ${ }^{3}$ IBM Research, Zurich Research Laboratory, CH-8803 Rüschlikon, Switzerland
}

(Received 2 January 2007; revised manuscript received 16 April 2007; published 12 July 2007)

\begin{abstract}
The mode spectrum of micrometer-sized ferromagnetic Permalloy disks, exhibiting a vortex ground state, is investigated by means of time-resolved scanning Kerr microscopy. The temporal evolution of the magnetization is probed after application of a fast in-plane field pulse. The lowest order azimuthal mode, a mode with only one diametric node, splits into a doublet as the disk diameter decreases. Theoretical models show that this splitting is a consequence of the interaction of the mode with the gyrotropic motion of the vortex core. Our experiments and micromagnetic simulations confirm that by removing the vortex core from the disk, the mode splitting vanishes.
\end{abstract}

DOI: 10.1103/PhysRevB.76.014416

PACS number(s): 75.75.+a, 75.40.Gb

The spectra and spatial structure of spin-wave modes in small ferromagnetic elements characterized by an almost flux-closed (stray field free) magnetization state have been thoroughly investigated in recent years. Among the magnetic elements which have been investigated in detail, one may find squares, ${ }^{1-3}$ rings, ${ }^{4,5}$ stripes, ${ }^{6-8}$ and circular disks. ${ }^{9-11}$ In some recent papers, the attempts to manipulate the structure of spin-wave modes in confined magnetic elements by modification of the elements' physical properties have been reported. ${ }^{10-12}$ A particular attention has been paid to diskshaped elements due to their simplicity. The response of these elements to the external excitation with pulsed and continuous magnetic fields has been studied both experimentally and theoretically. ${ }^{10,11,13,14}$

In general, two types of dynamic excitations can be found in these cylindrically shaped elements that exist in the vortex ground state. The first type, known as a gyrotropic mode, ${ }^{1,15,16}$ is the oscillatory motion of the vortex core itself. In the absence of an external bias field, the vortex core is located at the center of the disk. After the application of an in-plane magnetic field pulse, the vortex is displaced from the center. While the system relaxes toward its equilibrium state, the vortex gyrates around the disk center. With the disk diameters in the micrometer range, the frequencies of this gyrotropic mode lie in the subgigahertz range. In the experiments described below, the accessible time range was about $3.4 \mathrm{~ns}$ and, therefore, the gyrotropic mode could not be observed.

The second type of modes, known as magnetostatic modes, is the relatively high-frequency (several gigahertz) spin-wave excitations. These modes in the cylindrical geometry might have circular nodal lines (characterized by the radial index $n$ ) and diametric nodal lines (characterized by the azimuthal index $m$ ). In a recent paper, ${ }^{9}$ it has been shown that for disks having a large aspect ratio of diameter (e.g., several micrometers) to thickness (e.g., $20 \mathrm{~nm}$ ), the experimentally measured frequencies of these excitations can be well described by considering only the dipolar energy and pinned boundary conditions.

When the disk diameter is decreased, the influence of the vortex core and of the exchange interaction must be taken into account. In Ref. 11, the authors studied the excitation of spin-wave modes in small magnetic disks by in-plane and perpendicular field pulses. The effect of the vortex core polarization on the magnetostatic modes with one diametric node $(m= \pm 1)$ in similar small disks has been investigated in detail by Park and Crowell. ${ }^{10}$ In contrast to a recent paper ${ }^{4}$ where Permalloy rings with a large inner disk diameter of $850 \mathrm{~nm}$ were studied experimentally, in our experiments the inner disk diameter is small $(\approx 100 \mathrm{~nm})$. This allows us to uncover the influence of the vortex core on the spectrum of magnetostatic modes by comparing disks with and without the vortex core.

Standard disk samples (with the vortex core) were produced by the e-beam lithography and lift-off techniques and were placed on the center line of a 10- $\mu \mathrm{m}$-wide coplanar waveguide. For the measurement of the disks without the vortex core, the central region of the disks (a circular area of $100 \mathrm{~nm}$ diameter) was removed by means of a focused ion beam. Disks with different diameters ranging from 6 to $1 \mu \mathrm{m}$ and a constant thickness $L=15 \mathrm{~nm}$ were prepared.

Time-resolved scanning Kerr microscopy was used to monitor the temporal evolution of the $z$ component of the magnetization after the excitation with a short magnetic inplane field pulse. In this pump-probe technique, a Ti:sapphire laser generates short light pulses with a duration of a few hundred femtoseconds and a central wavelength of $800 \mathrm{~nm}$. In order to obtain high spatial resolution, a part of the light is frequency doubled to $400 \mathrm{~nm}$, serving as the probe beam. Pump and probe pulses can be delayed with respect to each other by means of an optical delay line. The experiments were performed in zero bias field. The magnetic field pulses were produced by an Auston switch, which has fast rise times in the picosecond range, allowing one to excite the precessional motion of the magnetization in the gigahertz range. Due to the weak excitation, the deflection of the magnetization vector from its ground state is very small $\left(<2^{\circ}\right)$. The excitation was gated with a frequency of several kilohertz and the signal was recorded using a lock-in technique. With this stroboscopic technique, only changes of the magnetization $M_{z}(x, y, t)-M_{z}(x, y, t=0)$ with respect to the ground state magnetization $M_{z}(x, y, t=0)$ are observed. In order to visualize the spatial structures of the excited modes, 

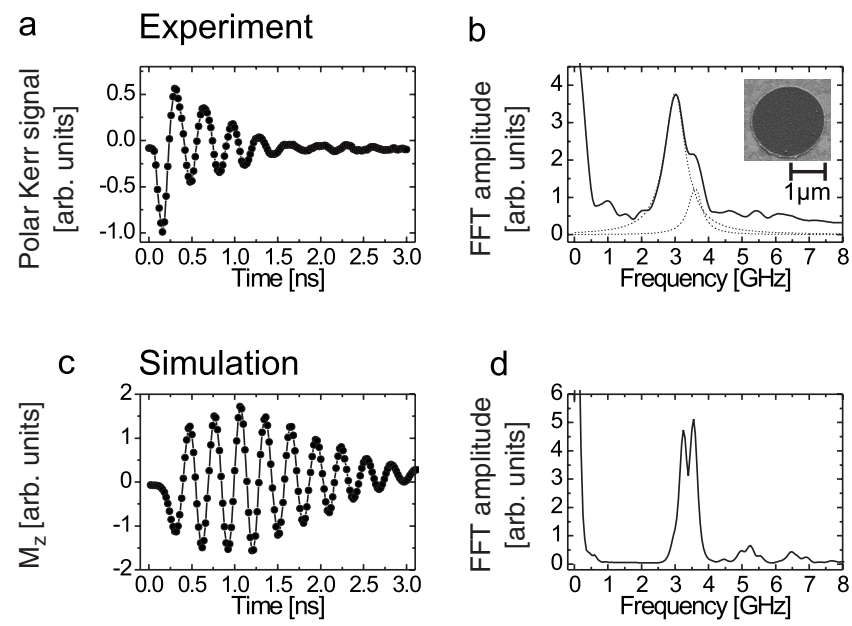

FIG. 1. Spatially averaged $z$ component of the magnetization of a disk (diameter $d=2 \mu \mathrm{m}$ and thickness $L=15 \mathrm{~nm}$ ) as a function of time obtained from (a) experiment and (c) simulation. Fourier transform amplitude for (b) experiment and (d) simulation. Two peaks appear in the experiment at 3.0 and $3.6 \mathrm{GHz}$, and in the simulations at 3.25 and $3.5 \mathrm{GHz}$. (e) The inset in (b) shows a scanning electron microscope image of the disk.

we recorded a sequence of spatially resolved images of the Kerr signal at different delay times with respect to the excitation pulse. A total of 150 images with a time step of 20 ps were taken. At first, the spatial average of the polar Kerr signal of the whole disk was calculated and plotted as a function of time. However, this total signal is very small when the local regions of nearly the same size oscillate with opposite phases. Therefore, in the following, the Kerr signal was averaged only over one side of the disk and plotted as a function of the delay time $t$.

In order to interpret our experimental findings, micromagnetic simulations using the commercially available LLG code $^{17}$ were performed. As parameters, we used saturation magnetization $M_{S}=800 \mathrm{kA} / \mathrm{m}$, exchange constant $A=13 \times 10^{-12} \mathrm{~J} / \mathrm{m}$, and damping constant $\alpha=0.01$. A cell size of $5 \times 5 \times 13 \mathrm{~nm}^{3}$ was chosen in the simulations in order to include the influence of the vortex core.

The spatial average of the $z$ component of the magnetization as a function of time elapsed after the application of the field pulse $H_{P}$ is plotted in Fig. 1. A decaying oscillation is found both in experiment and simulation. As different magnetic eigenmodes superimpose in a complicated way, the mode frequencies are not obvious at first glance. A Fourier transformation of the time domain data into the frequency domain allows one to determine the mode frequencies. We applied "zerofilling" and a Hamming window before the application of the Fourier transformation. A double peak structure with a major peak at $3.0 \mathrm{GHz}$ and a minor peak at $3.6 \mathrm{GHz}$ is found in the experiment [Fig. 1(b)], whereas two peaks of nearly equal amplitudes at 3.25 and $3.5 \mathrm{GHz}$ are obtained in the simulations [Fig. 1(d)]. The Fourier components of the exciting magnetic field pulse are probably responsible for the clearly different amplitudes of the fast Fourier transform peaks in the experiment [Fig. 1(b)]. A Fourier analysis of the current pulse taken with a high-bandwidth
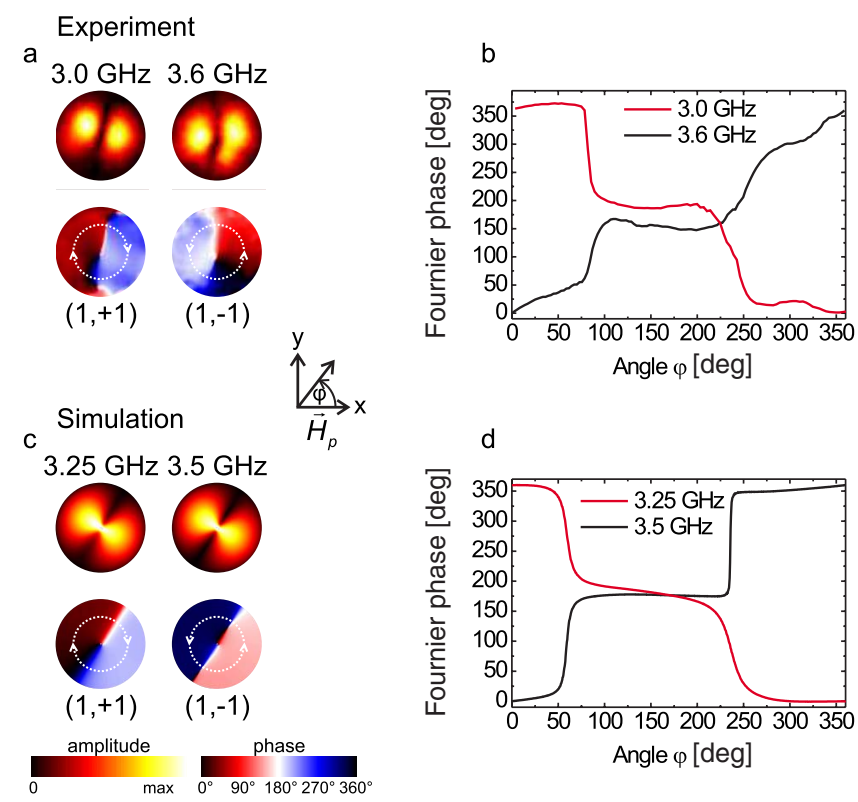

FIG. 2. (Color online). Spectral amplitude and phase images of the two azimuthal modes $(1,+1)$ and $(1,-1)$ from (a) experiment and (c) simulation. Only the first $1.5 \mathrm{~ns}$ were used to calculate the simulated images (c). [(b) and (d)] Scans along the circular paths of the disk as indicated by the dotted lines in (a) and (c). Both in experiment and simulation, the lower frequency mode rotates clockwise, while the higher frequency mode rotates counterclockwise.

sampling oscilloscope has shown that the Fourier amplitude of the exciting field pulses is approximately twice as large at $3 \mathrm{GHz}$ compared to $3.6 \mathrm{GHz}$. In addition, our experimental data can be well fitted by a superposition of two Lorentzianshaped curves with center frequencies of 3.0 and $3.6 \mathrm{GHz}$, as indicated by the two dotted lines in Fig. 1(b).

In order to obtain a deeper insight into the modal structure, we perform a local phase-sensitive Fourier transformation [Fig. 2(a)]. Here, for every point in space, a Fourier transformation is applied to the time domain data. The resulting data are reassembled to images in the frequency domain. This phase-sensitive technique allows one to directly visualize different magnetic modes since the Fourier transformation results in images of the amplitude and phase. Due to the cylindrical shape of the disks, modes with circular and diametric nodal lines can be observed and the eigenmodes can be labeled by two integers $(n, m), n$ counting the number of radial antinodes and $m$ counting the number of azimuthal nodes. Although higher order eigenmodes with more radial or azimuthal modes were observed in the experiment, we focus only on the lowest order azimuthal mode, which has the largest spectral weight. Due to the vortex ground state, the magnetization on the left side and on the right side of the disk is aligned antiparallel. Therefore the torque $\vec{M} \times \vec{H}_{P}$, which is exerted by the magnetic field pulse $H_{P}$, points in opposing directions, which becomes evident in the experiment. The spectral amplitude images in Fig. 2(a) correspond to the two strongest peaks in the Fourier spectrum and show two oscillating regions which are nearly equal in size. In addition, the phase images show no sharp nodal line between 
the left and right sides of the disk. The phase changes rather gradually from 0 to $360^{\circ}$ when the disk is traversed along the circular paths shown in Fig. 2(a). Moreover, at $3.0 \mathrm{GHz}$, the phase changes clockwise, while at $3.6 \mathrm{GHz}$, it varies counterclockwise, which is supported by the phase scans in Fig. 2(b). Both eigenmodes are traveling waves in the angular direction; i.e., their nodal lines are not fixed in space. The first one at $3.0 \mathrm{GHz}$ circulates clockwise, while the other one with $3.6 \mathrm{GHz}$ circulates counterclockwise. Therefore these modes are labeled $m= \pm 1$, indicating the sense of rotation (clockwise or counterclockwise).

Figure 1 shows that the frequency splitting of the modes in the experiment $\left(f_{S}=0.6 \mathrm{GHz}\right)$ is more than twice as large as in the simulation $\left(f_{S}=0.25 \mathrm{GHz}\right)$. The larger value in the experiment might be due to imperfections of the sample such as a slightly elliptical shape, growth induced magnetic anisotropy, or pinning. We would like to point out that these experimental results on the regular disks (with the vortex core) are in good qualitative agreement with the earlier experiments and simulations by Park and Crowell ${ }^{10}$ and Zhu et al. ${ }^{11}$

In Fig. 2(a), one can see that the nodal line of the modes is tilted by an angle of approximately $20^{\circ}$ with respect to the direction of the waveguide ( $y$ direction). This effect can be understood in the following way: two circulating modes with $m= \pm 1$ are excited at the same time and oscillate at slightly different frequencies, which leads to an interference effect. The superposition of these two waves having the same amplitude, opposite direction of circulation, and different frequencies results in a linearly polarized wave with a time dependent rotating nodal line. The period of this rotation is given by the frequency difference of the two modes, i.e., the frequency of the rotation corresponds to the frequency splitting $f_{S}$. In the simulation, the period of rotation should be about $1 / f_{S}=1 / 0.25 \mathrm{GHz}=4 \mathrm{~ns}$. Due to damping and the application of a Hamming window, the local Fourier transformation will most strongly emphasize the situation as it exists about $0.5 \mathrm{~ns}$ after the pulse excitation. One can therefore conclude that the nodal line should be tilted by an angle of about $\frac{0.5 \mathrm{~ns}}{4 \mathrm{~ns}}\left(360^{\circ}\right)=45^{\circ}$ with respect to the waveguide, in accordance with the simulated amplitude and phase images [Fig. 2(c)]. In the experiment, the observed tilt is smaller (only $20^{\circ}$ ) due to a combination of the damping, the Hamming window, and the magnitude of the splitting $f_{S}$. The Fourier transformation in the experiment emphasizes a time window centered 0.9 ns after the pump pulse. The larger frequency splitting of $0.6 \mathrm{GHz}$ corresponds to a rotation period of the nodal line of about $1.67 \mathrm{~ns}$. Therefore one can expect a tilt of $\frac{0.9 \mathrm{~ns}}{1.67 \mathrm{~ns}}\left(360^{\circ}\right) \approx 195^{\circ}$, corresponding to $180^{\circ}$ plus $15^{\circ}$, in reasonable agreement with the experimentally observed $20^{\circ}$.

In order to gain deeper insight into the influence of the vortex core on the modal spectrum, we also examined disks with the same diameters and thicknesses but with a circular hole of $100 \mathrm{~nm}$ diameter at the disk center. Figure 3 summarizes the results for a disk with a diameter of $2 \mu \mathrm{m}$. The clearly nonexponential decay of the Kerr signal in the experiment [Fig. 3(b)] may be mainly attributed to the shape of the excitation pulse, which has a fast rise time of several pico-
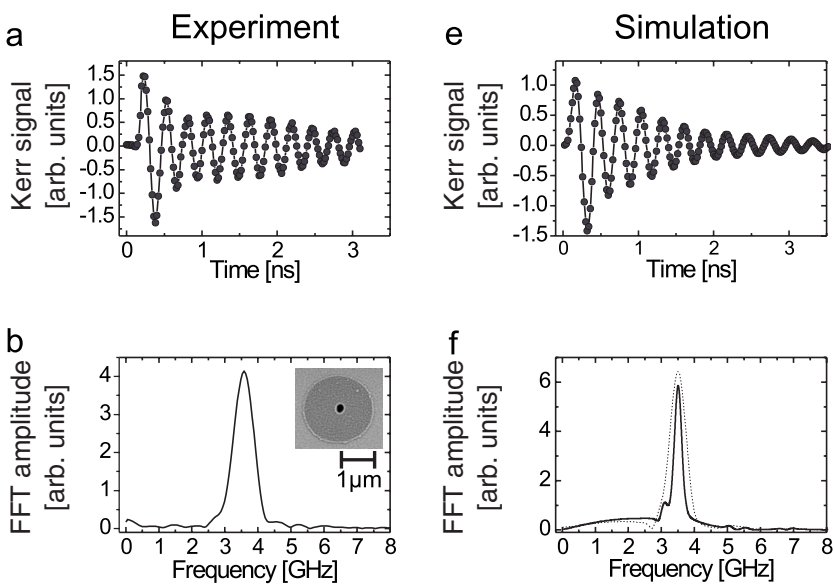

C
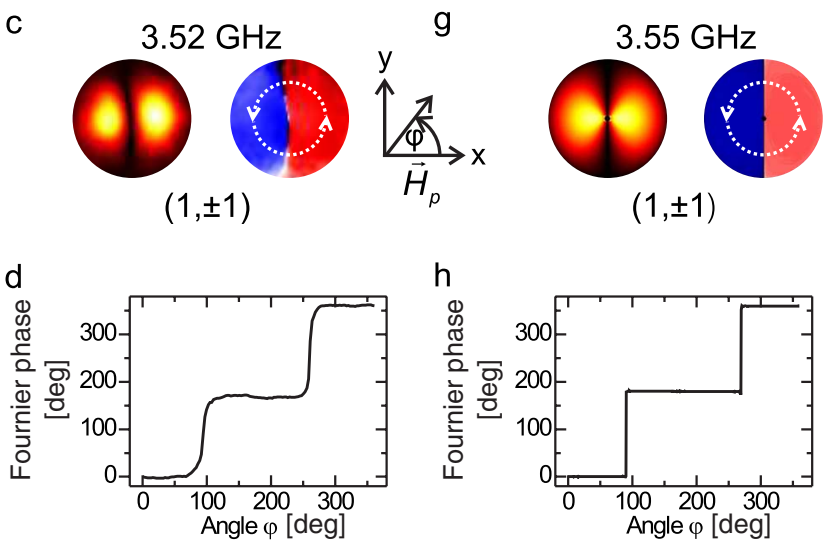

FIG. 3. (Color online) Results for a disk with a hole and diameter $d=2 \mu \mathrm{m}$ : data from experiment (left) and simulation (right). Spatially averaged $z$ component of the $[(a)$ and (e)] magnetization and $[(b)$ and (f)] Fourier transform amplitude. In the global Fourier amplitude of the experimental (simulated) data only a single peak is found at $3.5 \mathrm{GHz}(3.55 \mathrm{GHz})$. The small peak at approximately $3 \mathrm{GHz}$ in (f) can be associated with a mode which has two nodal lines in the azimuthal direction $(m=2)$. For a better comparison of the experimental and simulated data, the Fourier amplitude (f) was also computed for a limited time range of $3.4 \mathrm{~ns}$ (dashed line). In this case, the width of the peaks in experiment and simulation are comparable. [(c) and (g)] Local Fourier amplitude and phase images corresponding to the prominent peaks in the Fourier spectra. [(d) and (h)] The scans along the circular paths in the phase images (c) and $(\mathrm{g})$ reveal two jumps by $180^{\circ}$. Inset: The scanning electron microscope image shows the disk without vortex core. The central region of the disk, a circular area of $100 \mathrm{~nm}$ diameter, was removed by means of a focused ion beam.

seconds and a slow fall time of several hundreds of picoseconds. In addition, partial reflections of the current pulse at the ends of the coplanar waveguide may also contribute to the nonideal decay behavior. Since no interaction with the vortex core can take place, instead of a doublet structure, only a single peak at $3.5 \mathrm{GHz}$ is found in the global Fourier transform graphs in Figs. 3(b) and 3(f). The corresponding amplitude and phase images show two regions of nearly equal size, separated by a sharp nodal line and precessing with opposite phase. In contrast to the disk without a hole (Fig. 2), the phase does not change gradually in the angular 
direction, but jumps by $180^{\circ}$ as shown by the circular phase scans in Figs. 2(g) and 2(h). The two clockwise and counterclockwise circulating modes $(1,+1)$ and $(1,-1)$ are still present, even if the vortex core is removed. Without the interaction with the vortex core, both modes are degenerate, and therefore, have the same frequency. In contrast to the disk without a hole, the superposition of these two traveling modes with equal frequencies forms the spin-wave mode $(1, \pm 1)$ with a spatially fixed nodal line as observed in Figs. $3(\mathrm{c})$ and $3(\mathrm{~g})$. The tilt of the nodal line, which was observed for the nondegenerate modes in the regular disk without a hole [Fig. 2(a)], has disappeared. The nodal line is aligned exactly perpendicular to the pulse field direction $H_{P}$ and parallel to the coplanar waveguide.

Additional measurements with different disk diameters were performed in order to compare the mode frequencies and the size of the splitting to theoretical and experimental works by other groups. ${ }^{10,11,18}$ The frequency dependence of the first azimuthal mode $(1, \pm 1)$ is shown in Fig. 4 for both regular disks and disks with hole. The experimental data are compared with the numerical result obtained from the purely dipolar model in Ref. 9 and with the approximate analytical dipole-exchange model developed by Slavin et al. ${ }^{19}$

Good agreement between the models and the experiment is found when a thickness of $13 \mathrm{~nm}$ is used for the calculation of the theoretical values. The choice of this thickness is justified, since the thickness of the material was only determined with an error of $2 \mathrm{~nm}$. In addition, atomic force microscopy has shown that the sample surface has a rms roughness of about $2 \mathrm{~nm}$. Note that the frequency splitting of the azimuthal mode $(1,1)$ in Fig. 4(a), due to the interaction with the low-frequency gyrotropic mode, was not taken into account in both numerical and analytical calculations. The analytic approach ${ }^{19}$ results in the simple expression for the frequencies of both radial and azimuthal spin-wave modes of a magnetic ring or disk in a vortex ground state and is briefly discussed below.

We consider a circular ring element of thickness $L$ having internal radius $R_{1}$ and external radius $R_{2}$. The disk-shaped element of radius $R$ is approximated by a ring having $R_{2}=R$, and the inner radius equal to the radius of the vortex core $R_{1}=R_{\text {core }}=l_{\text {ex }}$, where $l_{\mathrm{ex}}=\sqrt{2 A / M_{s}^{2}}, M_{s}$ is the saturation magnetization, and $A$ is the exchange stiffness. In planar polar coordinates $(r, \phi)$, the static magnetization in the vortex state can be written as $\mathbf{M}=M_{s} \mathbf{e}_{\phi}$, and the ring becomes mathematically equivalent to a planar magnetic element uniformly magnetized in plane. To calculate the frequencies $\omega_{n m}$ of the spin-wave eigenmodes of such an element, we use a diagonal dipole-exchange dispersion equation for an infinite magnetic film of thickness $L$ uniformly magnetized in the film plane [see, e.g., Eq. (7) in Ref. 20], which was simplified and rewritten in polar coordinates for the case of zero bias magnetic field as

$$
\frac{\omega_{n m}^{2}}{\omega_{M}^{2}}=\frac{k_{r, n} L}{2} \frac{k_{r, n}}{\sqrt{k_{r, n}^{2}+k_{\phi, m}^{2}}}+\frac{2 A}{M_{s}^{2}}\left(k_{r, n}^{2}+k_{\phi, m}^{2}\right),
$$

where the quantized in-plane components of the mode wave numbers $k_{r, n}$ and $k_{\phi, m}$ are defined as

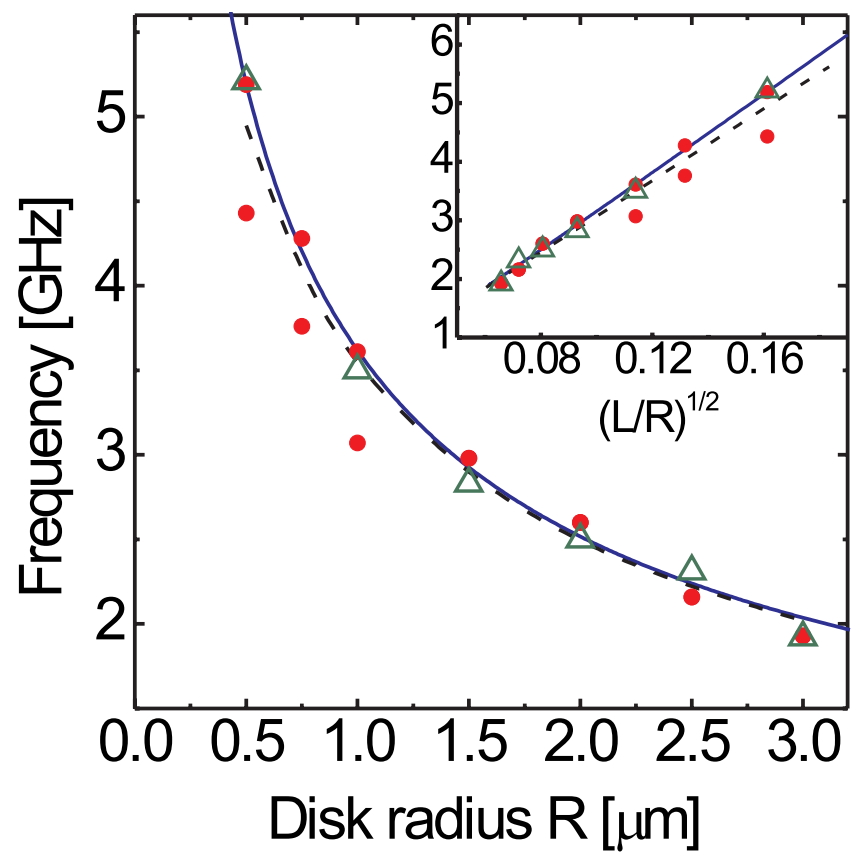

FIG. 4. (Color online) Size dependence of the frequency of the first azimuthal mode $(1,1)$. The experimentally obtained frequencies as a function of the disk radius $R$ for the full disks are represented by the filled circles $(\mathbf{)})$; the disks with a $100 \mathrm{~nm}$ hole are represented by the open triangles $(\triangle)$. The dashed and solid lines correspond to the theoretical calculations from Ref. 9 and from the dipole-exchange model [Eq. (1)], respectively, for a disk thickness $L=13 \mathrm{~nm}$. The inset shows the mode frequencies as a function of the square root of the aspect ratio $L / R$. A linear dependence of the frequencies is expected for the investigated size regime. The frequency error of the experimental data can be estimated by the time range $T=3.4 \mathrm{~ns}$, which corresponds to a frequency resolution of $1 / T \approx 0.3 \mathrm{GHz}$.

$$
\begin{gathered}
k_{r, n}=(n+1) \pi /\left(R_{2}-R_{1}\right), \\
k_{\phi, m}=m \ln \left(R_{2} / R_{1}\right) / \sqrt{R_{2}^{2}-R_{1}^{2}},
\end{gathered}
$$

with $n, m=0,1,2, \ldots ; \omega_{M}=4 \pi \gamma M_{s}$ and $\gamma$ is the gyromagnetic ratio.

The approximate dispersion Eq. (1) was obtained under the simplifying assumptions that $L /\left(R_{2}-R_{1}\right) \ll 1$ and $l_{\text {ex }}=\sqrt{2 A / M_{s}^{2}} \ll\left(R_{2}-R_{1}\right)$. The quantization condition (2) for the radial component $k_{r, n}$ was derived assuming very strong pinning of magnetization at the radial boundaries of the ring [which is reasonable for $L /\left(R_{2}-R_{1}\right) \ll 1$ (Ref. 21)], while a similar condition (3) for the azimuthal component $k_{\phi, m}$ was derived by imposing a periodic boundary condition in an infinitely narrow ring of radius $r$ and averaging the obtained $r$ dependent $k_{\phi, m}^{2}(r)=(m / r)^{2}$ over the interval $R_{1} \leqslant r \leqslant R_{2}$. Note that the obtained approximate dispersion equation takes into account not only dipole-dipole interaction but also exchange interaction, which is important for samples of smaller size or/and for modes with higher indices $n, m$.

It is clear from Fig. 4(a) that the approximate dipoleexchange analytical approach Eq. (1) and the dipolar numerical approach presented in Ref. 9 give good agreement with 
the experiment, but do not describe splitting of modes with $m=+1$ and $m=-1$.

Note that Eq. (1) explicitly shows the almost linear dependence of the mode frequency on $\sqrt{L / R}$, as demonstrated experimentally in the inset of Fig. 4. The same dependence was already observed in the analytic calculations of Novosad et al. $^{22}$

In Fig. 4, the mode frequencies of the disks without the vortex core are shifted upward with respect to the frequencies of the regular disks, which is in qualitative agreement with our simulations and the frequencies calculated with Eq. (1), for an inner disk diameter of $100 \mathrm{~nm}$. A simple explanation is that the additional inner disk boundary enhances the magnetic stiffness and thus leads to an increase of the mode frequencies. Furthermore, our experiments are also consistent with the simulations from Ref. 11 where the hole diameter is only $5 \mathrm{~nm}$ and the frequency lies perfectly in between the two mode frequencies of the disk without hole. In this case, the influence of the small inner disk boundary on the mode frequency is negligible. In agreement with our experimental results, the theoretical calculations by Zaspel and Ivanov $^{23}$ also find a slight increase of the frequencies of the azimuthal modes $m=0$ and $m=1$ with increasing inner radius for a fixed outer radius.

Up to now, only the theoretical calculations by Zaspel et al. ${ }^{18}$ allow one to calculate the splitting of the azimuthal mode $(1,1)$. This approach includes both the exchange interaction and the spin-wave scattering at the vortex core. Unfortunately, this model only provides a numerical solution. The frequency splitting in our experiments and simulations clearly exceeds the splitting from the calculations of Zaspel et al. ${ }^{18}$ but it is comparable with the experiments of other groups. ${ }^{10,11}$ Thus, a satisfying explanation for the discrepancy between experiment and theory for the magnitude of this splitting is still missing and calls for the refinement of the theoretical model.

In summary, we have investigated discrete spin-wave modes of disks in the flux-closed magnetization state with and without a vortex core at their center. Our experimental results confirm the possibility to control the magnetic response of simple magnetic elements by changing their shape. In addition, we have presented a simple dipole-exchange model that can adequately describe the spectrum of spinwave modes of disks and rings in the flux-closed state. Our experimental results concerning the splitting of the modes are in good agreement with micromagnetic simulations and can be explained qualitatively by the theoretical analysis of Zaspel and Ivanov.

Financial support by the priority program SPP1133 from DFG and by the MURI Grant No. W911NF-04-1-0247 from the U.S. Army Research Office is gratefully acknowledged.
${ }^{1}$ J. P. Park, P. Eames, D. M. Engebretson, J. Berezovsky, and P. A. Crowell, Phys. Rev. B 67, 020403(R) (2003).

${ }^{2}$ K. Perzlmaier, M. Buess, C. H. Back, V. E. Demidov, B. Hillebrands, and S. O. Demokritov, Phys. Rev. Lett. 94, 057202 (2005).

${ }^{3}$ B. V. Waeyenberge et al., Nature (London) 444, 461 (2006).

${ }^{4}$ X. Zhu, M. Malac, Z. Liu, H. Qian, V. Metlushko, and M. R. Freeman, Appl. Phys. Lett. 86, 262502 (2005).

${ }^{5}$ J. Podbielski, F. Giesen, and D. Grundler, Phys. Rev. Lett. 96, 167207 (2006).

${ }^{6}$ C. Mathieu et al., Phys. Rev. Lett. 81, 3968 (1998).

${ }^{7}$ J. P. Park, P. Eames, D. M. Engebretson, J. Berezovsky, and P. A. Crowell, Phys. Rev. Lett. 89, 277201 (2002).

${ }^{8}$ C. Bayer, J. P. Park, H. Wang, M. Yan, C. E. Campbell, and P. A. Crowell, Phys. Rev. B 69, 134401 (2004).

${ }^{9}$ M. Buess, T. P. J. Knowles, R. Höllinger, T. Haug, U. Krey, D. Weiss, D. Pescia, M. R. Scheinfein, and C. H. Back, Phys. Rev. B 71, 104415 (2005).

${ }^{10}$ J. P. Park and P. A. Crowell, Phys. Rev. Lett. 95, 167201 (2005).

${ }^{11}$ X. Zhu, Z. Liu, V. Metlushko, P. Grütter, and M. R. Freeman, Phys. Rev. B 71, 180408(R) (2005).

${ }^{12}$ R. L. Compton and P. A. Crowell, Phys. Rev. Lett. 97, 137202 (2006).

${ }^{13}$ M. Buess, R. Höllinger, T. Haug, K. Perzlmaier, U. Krey, D.
Pescia, M. R. Scheinfein, D. Weiss, and C. H. Back, Phys. Rev. Lett. 93, 077207 (2004).

${ }^{14}$ L. Giovannini, F. Montoncello, F. Nizzoli, G. Gubbiotti, G. Carlotti, T. Okuno, T. Shinjo, and M. Grimsditch, Phys. Rev. B 70, 172404 (2004).

${ }^{15}$ K. Y. Guslienko, B. A. Ivanov, V. Novosad, Y. Otani, H. Shima, and K. Fukamichi, J. Appl. Phys. 91, 8037 (2002).

${ }^{16}$ S. B. Choe, Y. Acremann, A. Scholl, A. Bauer, A. Doran, J. Stöhr, and H. A. Padmore, Science 304, 420 (2004).

${ }^{17}$ http://llgmicro.home.mindspring.com

${ }^{18}$ C. E. Zaspel, B. A. Ivanov, J. P. Park, and P. A. Crowell, Phys. Rev. B 72, 024427 (2005).

${ }^{19}$ A. Slavin, S. Tacchi, and V. Tiberkevich, Abstracts of the International Conference on Magnetism (INTERMAG), 2006 (unpublished), Paper GE-02.

${ }^{20}$ K. Y. Guslienko, R. W. Chantrell, and A. N. Slavin, Phys. Rev. B 68, 024422 (2003).

${ }^{21}$ K. Y. Guslienko and A. N. Slavin, Phys. Rev. B 72, 014463 (2005).

${ }^{22}$ V. Novosad, M. Grimsditch, K. Y. Guslienko, P. Vavassori, Y. Otani, and S. D. Bader, Phys. Rev. B 66, 052407 (2002).

${ }^{23}$ C. E. Zaspel and B. A. Ivanov, J. Magn. Magn. Mater. 286, 366 (2005). 